\title{
Stable phantom-energy wormholes admitting conformal motions
}

\author{
Peter K.F. Kuhfittig* \\ *Department of Mathematics, Milwaukee School of Engineering, \\ Milwaukee, Wisconsin 53202-3109, USA
}

\begin{abstract}
It has been argued that wormholes are as good a prediction of Einstein's theory as black holes but the theoretical construction requires a reverse strategy, specifying the desired geometric properties of the wormhole and leaving open the determination of the stress-energy tensor. We begin by confirming an earlier result by the author showing that a complete wormhole solution can be obtained by adopting the equation of state $p=\omega \rho$ and assuming that the wormhole admits a one-parameter group of conformal motions. The main purpose of this paper is to use the assumption of conformal symmetry to show that the wormhole is stable to linearized radial perturbations whenever $-1.5<\omega<-1$.

PAC numbers: 04.20.Jb, 04.20.-q, 04.20.Gz
\end{abstract}

\section{Introduction}

Wormholes are handles or tunnels in spacetime linking widely separated regions of our own Universe or different universes altogether [1]. While one could argue that wormholes are as good a prediction of Einstein's theory as black holes, the fact remains that a wormhole can only be held open by violating the null energy condition, which states that the stress-energy tensor $T_{\alpha \beta}$ must obey the condition $T_{\alpha \beta} k^{\alpha} k^{\beta} \geq 0$ for all null vectors [1]. Renewed interest in the subject of wormhole physics is due in part to the discovery that our Universe is undergoing an accelerated expansion [2, 3], i. e., $\ddot{a}>0$ in the Friedmann equation $\ddot{a} / a=-\frac{4 \pi}{3}(\rho+3 p)$. (Our units are taken to be those in which $G=c=1$.) The acceleration is caused by a negative pressure dark energy with equation of state $p=\omega \rho$, $\omega<-\frac{1}{3}$ and $\rho>0$. A value of $\omega<-\frac{1}{3}$ is required for an accelerated expansion, also referred to as quintessence dark energy. The value $\omega=-1$ corresponds to the existence of Einstein's cosmological constant [4]. Of particular interest is the case $\omega<-1$, usually referred to as phantom energy, which is slightly favored over quintessence observationally [5]. For the phantom-energy case, observe that $\rho+p<0$, in violation of the null energy

${ }^{*}$ kuhfitti@msoe.edu 
condition, thereby satisfying a fundamental requirement in wormhole physics. The only real objection that could be raised is that the notion of dark energy corresponds to a homogeneous distribution of matter, while wormhole spacetimes are necessarily inhomogeneous. Fortunately, the extension to spherically symmetric inhomogeneous spacetimes has been carried out. (See Ref. [6] for details.)

When Morris and Thorne [1] first proposed that wormholes may be actual physical objects, they described the wormhole by the static and spherically symmetric line element

$$
d s^{2}=-e^{2 \Phi(r)} d t^{2}+\frac{d r^{2}}{1-b(r) / r}+r^{2}\left(d \theta^{2}+\sin ^{2} \theta d \phi^{2}\right)
$$

Here $\Phi=\Phi(r)$ is called the redshift function, which must be everywhere finite to avoid an event horizon. The function $b=b(r)$ is called the shape function since it helps determine the spatial shape of the wormhole when viewed, for example, in an embedding diagram. The spherical surface $r=r_{0}$ is the throat of the wormhole and must satisfy the following conditions: $b\left(r_{0}\right)=r_{0}, b(r)<r$ for $r>r_{0}$, and $b^{\prime}\left(r_{0}\right)<1$, usually called the flare-out condition. This condition refers to the flaring out of the embedding diagram pictured in Ref. [1]. The flare-out condition can only be satisfied by violating the null energy condition. As already noted, in the present situation, this violation is a consequence of the phantom-energy background.

The Einstein field equations in the orthonormal frame, $G_{\hat{\mu} \hat{\nu}}=8 \pi T_{\hat{\mu} \hat{\nu}}$, yield the following simple interpretation for the components of the stress-energy tensor: $T_{\hat{t} \hat{t}}=\rho(r)$, the energy density, $T_{\hat{r} \hat{r}}=p_{r}$, the radial pressure, and $T_{\hat{\theta} \hat{\theta}}=T_{\hat{\phi} \hat{\phi}}=p_{t}$, the lateral pressure. For the theoretical construction of the wormhole, Morris and Thorne specified the functions $\Phi(r)$ and $b(r)$ to obtain the desired properties of the wormhole, thereby leaving the components of the stress-energy tensor dangling. This strategy would call for a search for those materials or fields that yield the required stress-energy tensor.

In a previous paper [19, the author had addressed this issue by introducing the barotropic equation of state $p=\omega \rho$, where $\omega<-1$ is the special case discussed above. By itself, this equation of state fails to produce a solution even if the energy density is known. The assumption of conformal symmetry fills the gap in the form of a complete wormhole solution.

The main purpose of this paper is to use the assumption of conformal symmetry to obtain conditions under which the wormhole is stable to linearized radial perturbations. The analysis leads to a physical interpretation of the conformal factor. We also need to recall briefly the definition of conformal Killing vectors, as well as the basic wormhole structure needed to perform the stability analysis. That is the topic of the next section.

A stability analysis of phantom-enery wormholes was also carried out by Lobo [8] but with very different assumptions, to be discussed later.

\section{Conformal Killing vectors and the shape function}

In this section we discuss the earlier assumption that our spacetime admits a one-parameter group of conformal motions. Recall that these are motions along which the metric tensor 
of a spacetime remains invariant up to a scale factor. This is equivalent to stating that there exists a set of conformal Killing vectors such that

$$
\mathcal{L}_{\xi} g_{\mu \nu}=g_{\eta \nu} \xi_{; \mu}^{\eta}+g_{\mu \eta} \xi_{; \nu}^{\eta}=\psi(r) g_{\mu \nu}
$$

where the left-hand side is the Lie derivative of the metric tensor and $\psi(r)$ is the conformal factor. The vector $\xi$ characterizes the conformal symmetry since the metric tensor $g_{\mu \nu}$ is conformally mapped into itself along $\xi$. It is generally agreed that the assumption of conformal symmetry has proved to be fruitful in numerous ways, not only leading to new solutions but also to new geometric and kinematical insights [9, 10, 11, 12, 13, 14, 15].

Exact solutions of traversable wormholes admitting conformal motions are discussed in Ref. [16] by assuming a noncommutative-geometry background. Two earlier studies assumed a non-static conformal symmetry [17, 18].

To discuss the consequences of the conformal-symmetry assumption, it turns out to be convenient to use the following form of the metric:

$$
d s^{2}=-e^{\nu(r)} d t^{2}+e^{\lambda(r)} d r^{2}+r^{2}\left(d \theta^{2}+\sin ^{2} \theta d \phi^{2}\right) .
$$

In particular, it is shown in Ref. [19] that

$$
e^{\nu}=C r^{2}
$$

while

$$
e^{\lambda}=\psi^{-2}
$$

Two of the Einstein field equations are

$$
8 \pi \rho=\frac{1}{r^{2}}\left(1-\psi^{2}\right)-\frac{\left(\psi^{2}\right)^{\prime}}{r}
$$

and

$$
8 \pi p_{r}=\frac{1}{r^{2}}\left(3 \psi^{2}-1\right)
$$

[See Ref. [19] for details.]

To obtain a wormhole solution, we start with the equation of state $p_{r}=\omega \rho, \omega<-1$, discussed in Sec. 1, and substitute Eqs. (6) and (7):

$$
\frac{1}{8 \pi} \frac{1}{r^{2}}\left(3 \psi^{2}-1\right)=\omega \frac{1}{8 \pi}\left[\frac{1}{r^{2}}\left(1-\psi^{2}\right)-\frac{\left(\psi^{2}\right)^{\prime}}{r}\right] .
$$

Simplifying, we have

$$
\left(\psi^{2}\right)^{\prime}+\frac{1}{r}\left(1+\frac{3}{\omega}\right) \psi^{2}=\frac{1}{r}\left(1+\frac{1}{\omega}\right) .
$$

This equation is linear in $\psi^{2}$ and can be readily solved to obtain

$$
\psi^{2}=\frac{\omega+1}{\omega+3}+D r^{-\frac{\omega+3}{\omega}},
$$


where $D$ is an arbitrary constant. By comparing Eqs. (1) and (3), we have, in view of Eq. (15),

$$
b(r)=r\left(1-e^{-\lambda}\right)=r\left(1-\psi^{2}\right) .
$$

To satisfy the condition $b\left(r_{0}\right)=r_{0}$, we must have $\psi^{2}\left(r_{0}\right)=0$, which becomes the initial condition for Eq. (9), thereby yielding $D$. The result is

$$
\psi^{2}=\frac{\omega+1}{\omega+3}-\frac{\omega+1}{\omega+3} r_{0}^{\frac{\omega+3}{\omega}} r^{-\frac{\omega+3}{\omega}} .
$$

The final forms are

$$
b(r)=r\left(1-\psi^{2}(r)\right)
$$

and

$$
\psi^{2}(r)=\frac{\omega+1}{\omega+3}\left(1-r_{0}^{\frac{\omega+3}{\omega}} r^{-\frac{\omega+3}{\omega}}\right) .
$$

Observe that $b\left(r_{0}\right)=r_{0}$, as noted above. A simple calculation now shows that since $\omega<-1, b^{\prime}\left(r_{0}\right)<1$. So the flare-out condition is met.

Our final observation is

$$
1-\frac{b(r)}{r}=\psi^{2}(r)
$$

to be used in Sec. 4 .

\section{Junction to an exterior vacuum solution}

We see from Eq. (4), $e^{\nu}=C r^{2}$, that the wormhole spacetime cannot be asymptotically flat. So the wormhole material must be cut off at some $r=a$ and joined (in the standard way) to an exterior Schwarzschild solution

$$
d s^{2}=-\left(1-\frac{2 M}{r}\right) d t^{2}+\frac{d r^{2}}{1-2 M / r}+r^{2}\left(d \theta^{2}+\sin ^{2} \theta d \phi^{2}\right) .
$$

Referring now to line element (11), we first note that $M=\frac{1}{2} b(a)$. So for $e^{\nu}=C a^{2}$, we have $C a^{2}=1-2 M / a$ and the integration constant becomes

$$
C=\frac{1}{a^{2}}\left(1-\frac{b(a)}{a}\right)
$$

thereby completing the wormhole solution. The junction surface plays an important role in the stability analysis in the next section.

\section{Stability analysis}

Our first task in this section is to study the stresses on the junction surface leading to the stability criterion. To that end, let us recall the Lanczos equations [20]

$$
\sigma=-\frac{1}{4 \pi} \kappa_{\theta}^{\theta}
$$


and

$$
\mathcal{P}=\frac{1}{8 \pi}\left(\kappa_{\tau}^{\tau}+\kappa_{\theta}^{\theta}\right)
$$

where $\kappa_{i j}=K_{i j}^{+}-K_{i j}^{-}$and $K_{i j}$ is the extrinsic curvature. According to Ref. [20],

$$
\kappa_{\theta}^{\theta}=\frac{1}{a} \sqrt{1-\frac{2 M}{a}}-\frac{1}{a} \sqrt{1-\frac{b(a)}{a}} .
$$

So by Eq. (17),

$$
\sigma=-\frac{1}{4 \pi a}\left(\sqrt{1-\frac{2 M}{a}}-\sqrt{1-\frac{b(a)}{a}}\right) .
$$

In view of the assumption $M=\frac{1}{2} b(a)$ in the previous section, one could reasonably expect that $\sigma=0$. However, part of the junction formalism is to assume that the junction surface $r=a$ is an extremely thin surface having a nonzero density. The mass of the shell is therefore given by

$$
m_{s}=4 \pi a^{2} \sigma=-a\left(\sqrt{1-\frac{2 M}{a}}-\sqrt{1-\frac{b(a)}{a}}\right) .
$$

Moreover, given that $\frac{1}{2} b(a)$ is the total mass inside a sphere of radius $a$, we see that if $m_{s}<0$, then $M<\frac{1}{2} b(a)$ and if $m_{s}>0$, then $M>\frac{1}{2} b(a)$.

To perform the stability analysis, we make the usual assumption that the junction surface is a function of proper time $\tau$ moving about some equilibrium position $a=a_{0}$. Following Lobo [8], the density takes on the form

$$
\sigma=-\frac{1}{4 \pi a}\left(\sqrt{1-\frac{2 M}{a}+\dot{a}^{2}}-\sqrt{1-\frac{b(a)}{a}+\dot{a}^{2}}\right),
$$

where $\dot{a}=d a / d \tau$.

To obtain the stability criterion, one starts by rearranging Eq. (22), namely

$$
\sqrt{1-\frac{2 M}{a}+\dot{a}^{2}}=\sqrt{1-\frac{b(a)}{a}+\dot{a}^{2}}-4 \pi a \sigma,
$$

to obtain the "equation of motion"

$$
\dot{a}^{2}+V(a)=0
$$

where $V(a)$ is the potential. It is a straightforward exercise to show that

$$
V(a)=1-\frac{M+b(a) / 2}{a}-\frac{m_{s}^{2}}{4 a^{2}}-\frac{(M-b(a) / 2)^{2}}{m_{s}^{2}} .
$$

The time-dependent radius allows us to study the effect of a radial perturbation around the static solution $a=a_{0}$. Again following Ref. [8], this requires linearizing around $a=a_{0}$ by considering the Taylor expansion of $V(a)$ about $a=a_{0}$ :

$$
V(a)=V\left(a_{0}\right)+V^{\prime}\left(a_{0}\right)\left(a-a_{0}\right)+\frac{1}{2} V^{\prime \prime}\left(a_{0}\right)\left(a-a_{0}\right)^{2}+\text { higher-order terms. }
$$


To meet the linearized stability criterion, we must have $V\left(a_{0}\right)=0$ and $V^{\prime}\left(a_{0}\right)=0$, while $V^{\prime \prime}\left(a_{0}\right)>0$.

The question arises in what sense these conditions are met and how to make best use of Eq. (24). To address this issue, consider the last term, denoted by $L^{2}$, i. e.,

$$
L=\frac{M-\frac{1}{2} b(a)}{-a\left(\sqrt{1-\frac{2 M}{a}}-\sqrt{1-\frac{b(a)}{a}}\right)} .
$$

After rationalizing the denominator and simplifying, we obtain

$$
L=\frac{M-\frac{1}{2} b(a)}{2 M-b(a)}\left(\sqrt{1-\frac{2 M}{a}}+\sqrt{1-\frac{b(a)}{a}}\right)=\frac{1}{2}\left(\sqrt{1-\frac{2 M}{a}}+\sqrt{1-\frac{b(a)}{a}}\right) .
$$

Since the equilibrium position $a=a_{0}$ refers to the junction surface where $2 M=b(a)$, we obtain $L=\sqrt{1-b\left(a_{0}\right) / a_{0}}$. The result is

$$
V\left(a_{0}\right)=1-\frac{b\left(a_{0}\right)}{a_{0}}-\frac{m_{s}^{2}}{4 a_{0}^{2}}-\left(\sqrt{1-\frac{b\left(a_{0}\right)}{a_{0}}}\right)^{2},
$$

which does indeed lead to $V\left(a_{0}\right)=0$ since $m_{s}=0$. Now, as noted earlier, part of the junction formalism is to assume that for a thin shell, $m_{s}$ cannot be zero. So $V\left(a_{0}\right)=0$ must be viewed as the dividing line between $m_{s}<0\left(2 M<b\left(a_{0}\right)\right)$ and $m_{s}>0\left(2 M>b\left(a_{0}\right)\right)$, implying $V\left(a_{0}\right)$ is equal to zero only in the limit as $\frac{1}{2} b\left(a_{0}\right) \rightarrow M$. The same holds for $V^{\prime}\left(a_{0}\right)=0$.

The implication is that the dynamic analysis leading to $V\left(a_{0}\right)=0$ and $V^{\prime}\left(a_{0}\right)=0$ requires the use of Eq. (21), thereby making $m_{s}$ a variable quantity. But once a particular junction surface $r=a$ has been chosen, $m_{s}$ is necessarily fixed at some positive or negative value. So we are going to make the following assumptions: to make use of Eq. (24), we assume that Eq. (21) is no longer needed and that $m_{s}^{2}$ is a small constant. Given that $b(a) \approx 2 M$, these assumptions lead at once to the approximation

$$
V(a) \approx 1-\frac{b(a)}{a}
$$

for any junction surface $r=a$. Moreover, the approximation can be naturally connected to the conformal symmetry. In other words, by Eq. (15),

$$
1-\frac{b(a)}{a}=\psi^{2}(a)
$$

So $\psi^{2}$ can be interpreted physically as an approximation of the potential:

$$
V(a) \approx \psi^{2}(a) .
$$

From Eq. (14), we now get

$$
V^{\prime \prime}\left(a_{0}\right)=\frac{d^{2}}{d a^{2}} \psi^{2}\left(a_{0}\right)=-\frac{\omega+1}{\omega} \frac{2 \omega+3}{\omega} r_{0}^{\frac{\omega+3}{\omega}} a_{0}^{-\frac{\omega+3}{\omega}-2} .
$$


Since $\omega<-1$, we conclude that $V^{\prime \prime}\left(a_{0}\right)>0$ only if $\omega>-1.5$. It follows that our wormhole is stable to linearized radial perturbations whenever

$$
-1.5<\omega<-1
$$

The conclusion is independent of the shape function and the junction surface. Lobo's earlier study [8] assumed a specific shape function but no conformal symmetry. Instead, letting $\omega=-2$, a typical value, the wormhole was found to be stable for a wide range of values of the radius of the junction surface.

\section{Conclusion}

For the theoretical construction of a traversable wormhole, Morris and Thorne proposed the following reverse strategy: specify the desired geometric properties of the wormhole, while leaving open the determination of the stress-energy tensor. The first part of this paper confirms an earlier result [19] stating that a complete wormhole solution can be obtained by adopting the equation of state $p=\omega \rho, \omega<-1$, and assuming that the wormhole admits a one-parameter group of conformal motions. The main purpose of this paper is to use the assumption of conformal symmetry to show that the wormhole is stable to linearized radial perturbations whenever $-1.5<\omega<-1$. The analysis also yielded a physical interpretation of the conformal factor in terms of the potential.

\section{References}

[1] M.S. Morris and K.S. Thorne, Amer. J. Phys. 56, 395 (1988).

[2] A.G. Riess et al., Astron. J. 116, 1009 (1998).

[3] S.J. Perlmutter et al., Astroph. J. 517, 565 (1999).

[4] M. Carmeli, arXiv: astro-ph/0111259.

[5] T.T. Huang, P.X. Wu, and H.W. Yu, Science China 53, 562 (2010).

[6] S.V. Sushkov, Phys. Rev. D 71, 043520 (2005).

[7] P.K.F. Kuhfittig, Annals of Physics, 355, 115 (2015).

[8] F.S.N. Lobo, Phys. Rev. D, 71, 124002 (2005).

[9] L. Herrera and J. Ponce de León, J. Math. Phys. 26, 778 (1985).

[10] L. Herrera and J. Ponce de León, J. Math. Phys. 26, 2018 (1985).

[11] R. Maartens and C.M. Mellin, Class. Quant. Grav. 13, 1571 (1996).

[12] M. Mars and J.M.M. Senovilla, Class. Quant. Grav. 10, 1633 (1993). 
[13] S. Ray, A.A. Usmani, F. Rahaman, M. Kalam, and K. Chakraborty, Ind. J. Phys. 82, 1191 (2008).

[14] F. Rahaman, M. Jamil, M. Kalam, K. Chakraborty, and A. Ghosh, Astrophys. Space Sci. 325, 137 (2010).

[15] F. Rahaman, S. Ray, I. Karar, H.I. Fatima, S. Bhowmick, and G.K. Ghosh, arXiv: 1211.1228 [gr-qc].

[16] F. Rahaman, S. Ray, G.S. Khadekar, P.K.F. Kuhfittig, and I. Karar, Int. J. Theor. Phys. 54, 699 (2015).

[17] C.G. Böhmer, T. Harko, and F.S.N. Lobo, Phys. Rev. D 76, 084014 (2007).

[18] C.G. Böhmer, T. Harko, and F.S.N. Lobo, Class. Quant. Grav. 25, 075016 (2008).

[19] P.K.F. Kuhfittig, Eur. Phys. J. C 75, 357 (2015).

[20] F.S.N. Lobo, Class. Quant. Grav. 21, 4811 (2004). 